with breast-conserving surgery for DCIS, to determine the influence of multifocality on local recurrence.

Among the study population, 310 patients were treated with breast-conserving surgery alone, whereas 305 received breast-conserving surgery and radiotherapy. Multivariate analysis revealed multifocality to be the strongest predictor of local recurrence (hazard ratio 1.8, 95\% Cl 1.2-2.8). Notably, radiotherapy was associated with a decreased risk of local recurrence. When the population was considered as a whole, there was no significant difference in the rate of local recurrence between women with multifocal DCIS (16.3\%) and women with unifocal disease (13.4\%; $P=0.25)$. Analyses stratified according to treatment group, however, revealed a detrimental effect of multifocality in those patients who had not received radiotherapy. In the surgery-only group, the recurrence-free survival rate at 10 years was $59 \%$ for women with multifocal DCIS compared with $80 \%$ for women with unifocal disease $(P=0.02)$. The corresponding recurrence-free survival rates for women who received both surgery and radiotherapy were $81 \%$ and $84 \%$, respectively $(P=0.40)$.

The authors conclude that breast-conserving surgery is a feasible treatment option for women with multifocal DCIS, provided adjuvant radiotherapy is administered.

Original article Rakovitch E et al. (2007) Significance of multifocality in ductal carcinoma in situ: outcomes of women treated with breast-conserving therapy. J Clin Oncol 25: 5591-5596

\section{Tissue-microarray study supports change to guidelines for assessment of HER2 expression}

The ASCO/College of American Pathologists guidelines for the interpretation of the companion diagnostic for trastuzumab have recently been amended to try to address the problem of tumor heterogeneity. The guidelines now state that $30 \%$ of the cells that represent the carcinoma must show positive immunohistochemical staining for human epidermal growth factor 2 (HER2) for the tumor to be considered HER2 positive (i.e. IHC3+). The threshold was previously $10 \%$.

Moeder et al. have conducted tissue-microarray studies to assess the levels of heterogeneity in the expression of three biomarkers-estrogen receptor, progesterone receptor and HER2 - in 669 breast-tumor samples. The researchers examined four to five separate tissuemicroarray cores for each tumor sample and assessed heterogeneity by linear regression analysis. Although all three biomarkers showed some heterogeneity between cores, the correlation coefficients were all in the range 0.7-0.8. Minimum, average and maximum scores for each marker were calculated for each patient and the prognostic importance of these scores determined. For the estrogen receptor, the maximum score was most prognostic of good outcome. By contrast, the minimum HER2 score was most prognostic for poor outcome and most predictive of response to trastuzumab. These results support the recent alteration to the guidelines for assessment of HER2 expression but suggest that they have not gone far enough. Furthermore, the data indicate that the degree of uniformity required for the most accurate prediction of biological impact varies according to the biomarker.

Original article Moeder CB et al. (2007) Quantitative justification of the change from $10 \%$ to $30 \%$ for human epidermal growth factor receptor 2 scoring in the American Society of Clinical Oncology/College of American Pathologists Guidelines: tumor heterogeneity in breast cancer and its implications for tissue microarray-based assessment of outcome. J Clin Oncol 25: 5418-5425

\section{Cyclophosphamide and dexamethasone as induction therapy for multiple myeloma}

In patients with multiple myeloma, combined vincristine, doxorubicin and dexamethasone (VAD) is the most frequently used induction therapy before autologous stem-cell transplantation (ASCT); however, toxicities related to VAD have been described, and administration of this therapy requires a central venous access. A study by Mellqvist et al. has assessed the efficacy and safety of combined bolus-dose cyclophosphamide and pulse-dose dexamethasone (Cy-Dex) as initial treatment for patients with myeloma.

The study included 314 patients with newly diagnosed disease, who were randomized to receive either three courses of VAD $(n=156)$ or two courses of Cy-Dex $(n=158)$ before ASCT. In total, $86 \%$ of patients allocated VAD and $87 \%$ of patients allocated Cy-Dex completed their course of chemotherapy and underwent ASCT. 